\title{
FINDING SIMILARITIES IN LASER MICROMETERS' SCANNED DATA USING CROSS-CORRELATION
}

\author{
J. Czebe* , P. Šuránek ${ }^{* *}$, J. Tůma***, D. Fojtík ${ }^{\dagger}$
}

\begin{abstract}
This paper demonstrates a signal processing approach designed to overlay data sets measured using two optical micrometers. Two sensors are used in order to measure object's shape, but measured signals are relatively shifted, because it is impossible to mount sensors into the same position. Correlation method are applied to measurement data and the shift between these sets are evaluated.
\end{abstract}

Keywords: signal processing, cross-covariance, optical micrometer, laser.

\section{Introduction}

Optical micrometres are sensors widely used for measuring dimensions of objects. Sensors consists of a transmitter and a receiver. The transmitter emits laser beam region to the receiver and when the measured object is placed between these two parts, it produces a shadow, which is detected by the receiver (Jones 1976). Micrometres can be used for the detection of the object's edge position (Widmaier 2011), for the measurement object's size (Prieur 2010), for the measurement of the gap and other applications (Riftek 2018). The aim of this contribution is to describe the procedure of processing two signals from optical sensors. These sensors measure a shape of an experimental object. Two signals are relatively shifted because mentioned sensors are mounted in different positions and a method using a correlation is described in order to find a shift between these signals.

\section{Description of an experiment}

An experimental object is fastened to the linear drive and is translating along the $\mathrm{z}$ axis. This movement is measured using an encoder. Laser micrometers measure the edge in $x$ axis and data is collected as a set of points in $z, x$ plane. Data from all sensors are acquired by micro-controller unit (MCU) and are deposited in a computer for further signal processing.
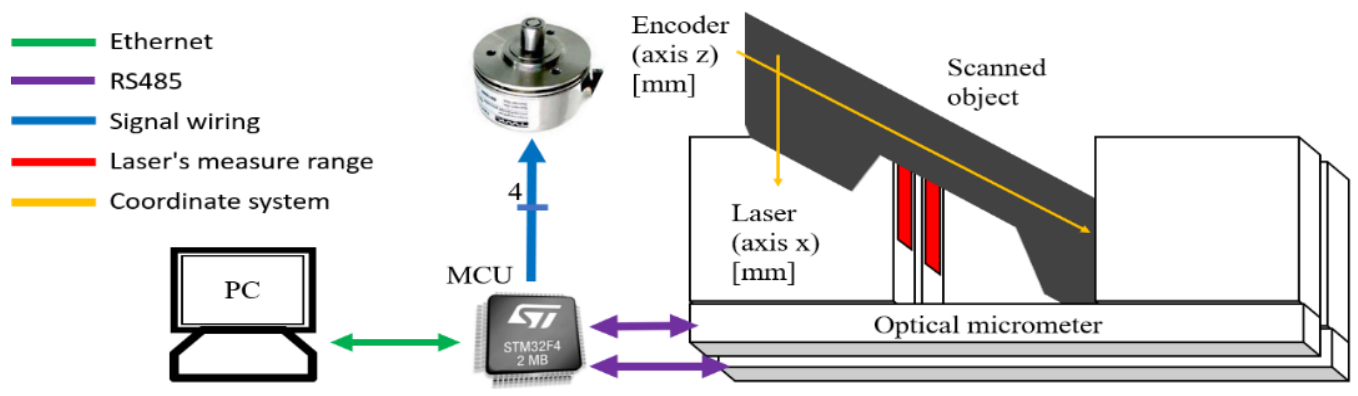

Fig. 1: Measurement chain

Ing. Jiří Czebe: Department of Control Systems and Instrumentation, Faculty of Mechanical Engineering, VSB - Technical University of Ostrava, Ostrava, Czech Republic; CZ, jiri.czebe@ vsb.cz

** Ing. Pavel Šuránek, PhD.: Department of Control Systems and Instrumentation, Faculty of Mechanical Engineering, VSB Technical University of Ostrava, Ostrava, Czech Republic; CZ, pavel.suranek@ vsb.cz

*** Prof. Ing. Jiří Tůma, CSc.: Department of Control Systems and Instrumentation, Faculty of Mechanical Engineering, VSB Technical University of Ostrava, Ostrava, Czech Republic; CZ, jiri.tuma@vsb.cz

$\dagger \quad$ Ing. David Fojtík, PhD.: Department of Control Systems and Instrumentation, Faculty of Mechanical Engineering, VSB Technical University of Ostrava, Ostrava, Czech Republic; CZ, david.fojtik@vsb.cz 
MCU collects raw data from lasers ( $x$-axis) and encoder ( $z$-axis), performs data filtering via median ( $x$ axis) and mean ( $z$-axis). Each measured point is created from a set of raw points belonging to circle range defined as dot pitch. MCU checks each new raw point: if a distance between a first point and a new one is greater than pre-set dot pitch, then MCU performs a calculation of mean and median from previously measured raw points, and the last one is considered as a first raw point of next circle range.

Figure 2 illustrates achieved results on test data (measured points) using the mentioned process.
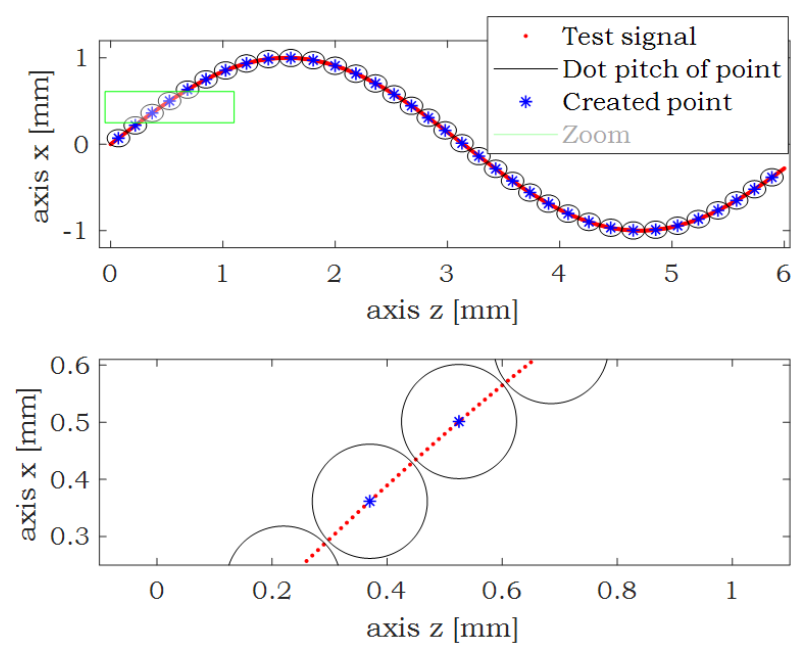

Fig. 2: Creation of measured points

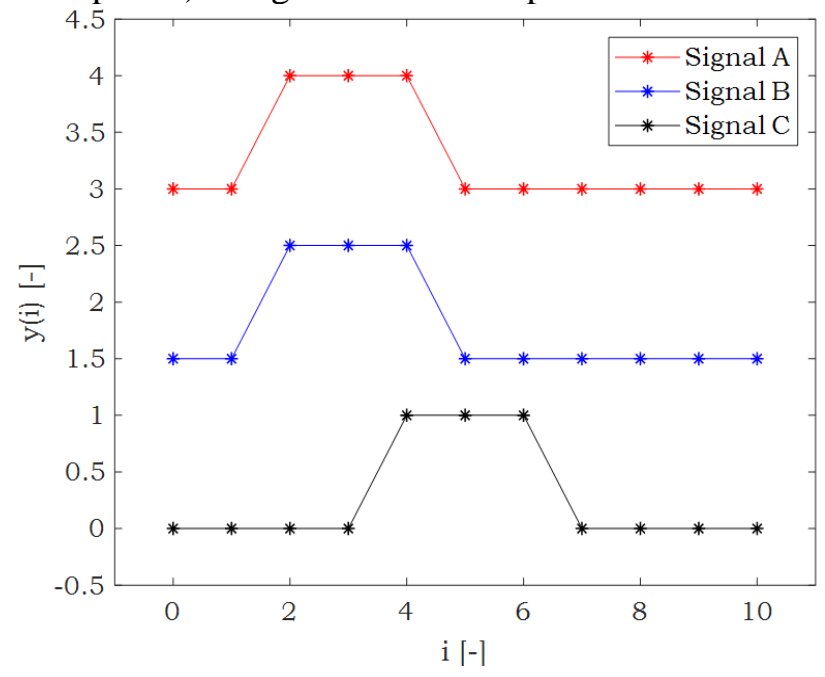

Fig. 3: Explanation of a correlation

\section{Theoretical background of signal processing}

The correlation is used to determines the change in one variable due to change in another variable. The correlation for two signal vectors $A, B$ using Pearson's linear correlation coefficient is defined:

$$
r_{A B}=\frac{\sum_{i=1}^{N}\left(A_{i}-\mu_{A}\right)\left(B_{i}-\mu_{B}\right)}{\sqrt{\sum_{i=1}^{N}\left(A_{i}-\mu_{A}\right) \sum_{i=1}^{N}\left(B_{i}-\mu_{B}\right)}}=\frac{\sum_{i=1}^{N}\left(A_{i}-\mu_{A}\right)\left(B_{i}-\mu_{B}\right)}{(N-1) \sigma_{A} \sigma_{B}}
$$

$N$ is number of elements in both vectors, $\mu_{A}$ is a mean value of a vector $A, \mu_{B}$ is a mean value of $B, \sigma_{A}$ is dispersion value of a vector $A$ and $\sigma_{B}$ is dispersion value of a vector $B$.

Figure 3 illustrates three signal vectors, where $m$ represents a lag between two signals. Signals $A$ and $B$ are positioned in a phase and signal $C$ is delayed by two samples. The correlation $(m=0)$ between vectors $A$ and $B$ is equal to 66, while the value of the correlation between $A$ and $C$ drops to 10 . Correlation is a useful tool to find shift between signals, results are highly affected by a form of input data if are in raw or standard (from each vector is subtracted its mean value).

The cross-correlation function enables to quantify correlation of two signals depended on the shift between these signals.

$$
r_{A B}(m)=\frac{\sum_{i=1}^{N}\left(A_{i+m}-\mu_{A}\right)\left(B_{i}-\mu_{B}\right)}{\sqrt{\sum_{i=1}^{N}\left(A_{i+m}-\mu_{A}\right) \sum_{i=1}^{N}\left(B_{i}-\mu_{B}\right)}}=\frac{\sum_{i=1}^{N}\left(A_{i}-\mu_{A+m}\right)\left(B_{i}-\mu_{B}\right)}{(N-1) \sigma_{A} \sigma_{B}}
$$

$m$ represents the shift (lag) in the number of samples.

Figure 4 illustrates three situations of computing the cross-correlation function of signals $A$ and $C$. When the shift of minus two samples is introduced, both signals are corresponding to each other and the highest value of cross-correlation function is obtained (see Figure 5). The original position of signals represents a zero shift. The last one has plus two samples with worst cross-correlation result.

\section{Processing of an experimental data}

Measured signals include a lot of invalid samples. These are caused by situations when the optical micrometer crosses a corner of the scanned object for example. It is needed to pick up horizontal sections from signals, which are supposed to be measured properly. These sections can be chosen as parts, where the absolute value of differentiation is low. Figure 6 depicts original measured data and selected parts. 


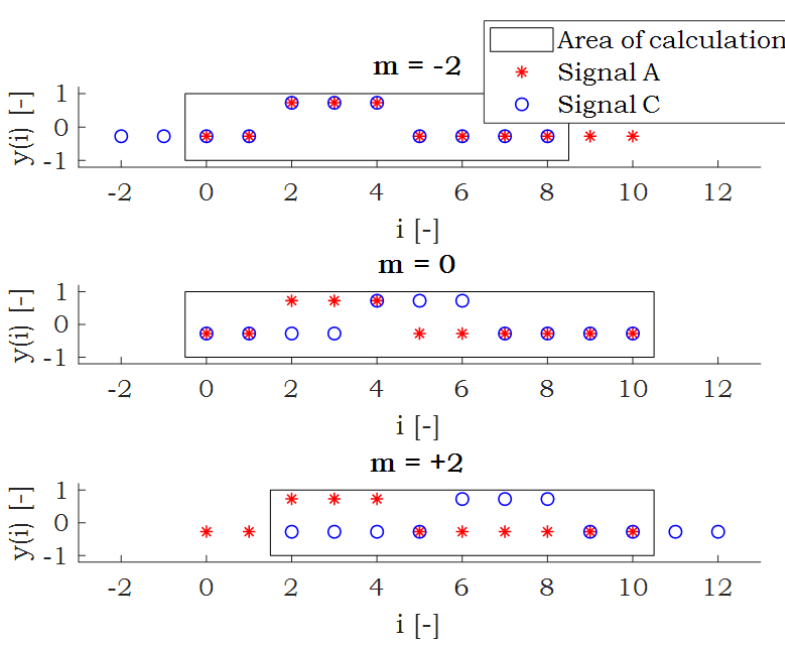

Fig. 4: Explanation of a cross-correlation
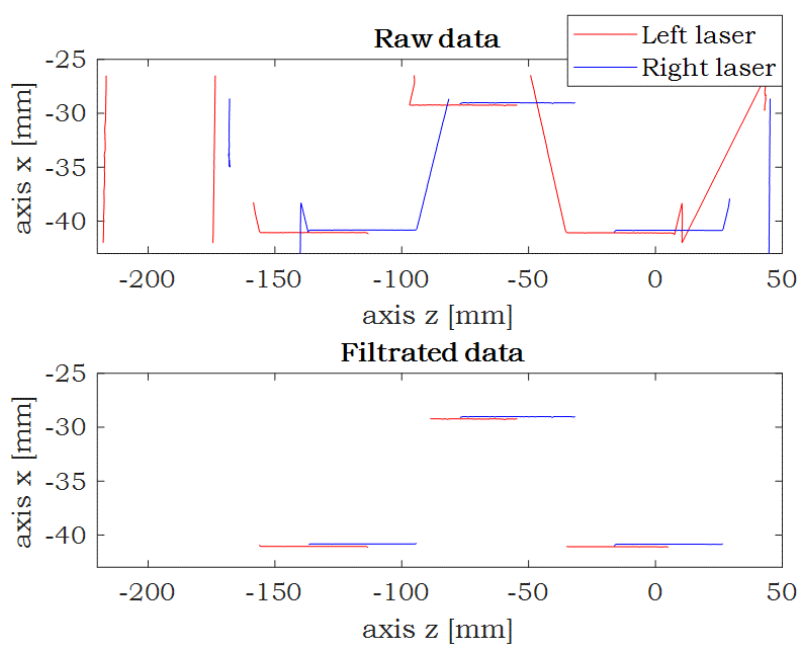

Fig. 6: Selection of data
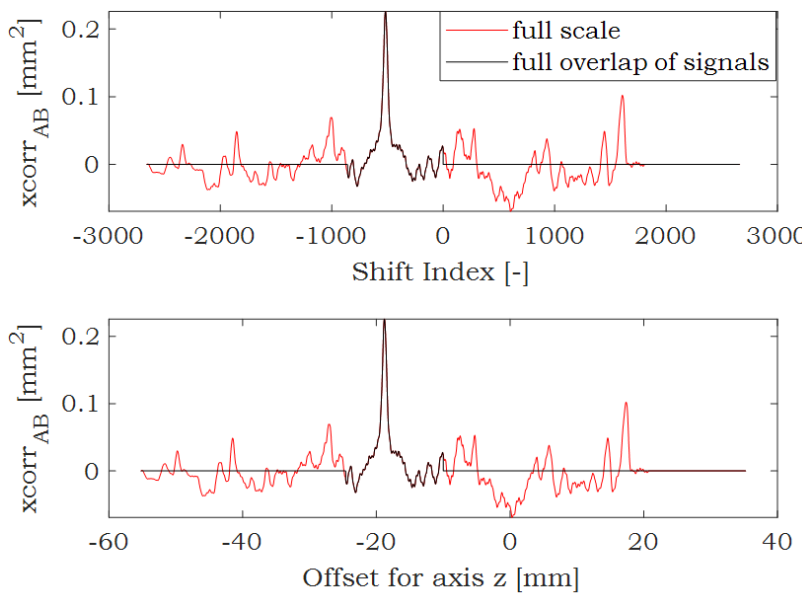

Fig. 8: Cross-Correlation
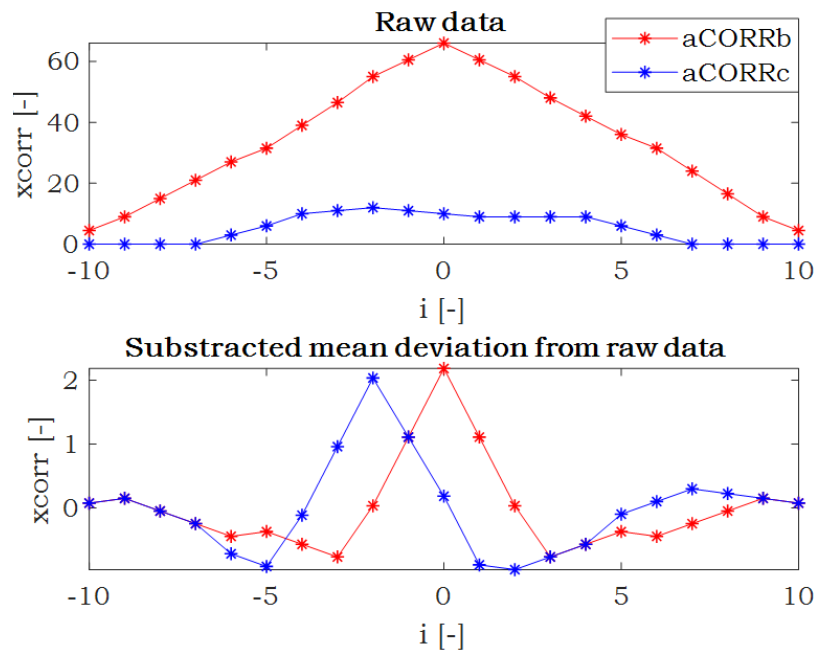

Fig. 5: Cross-correlation

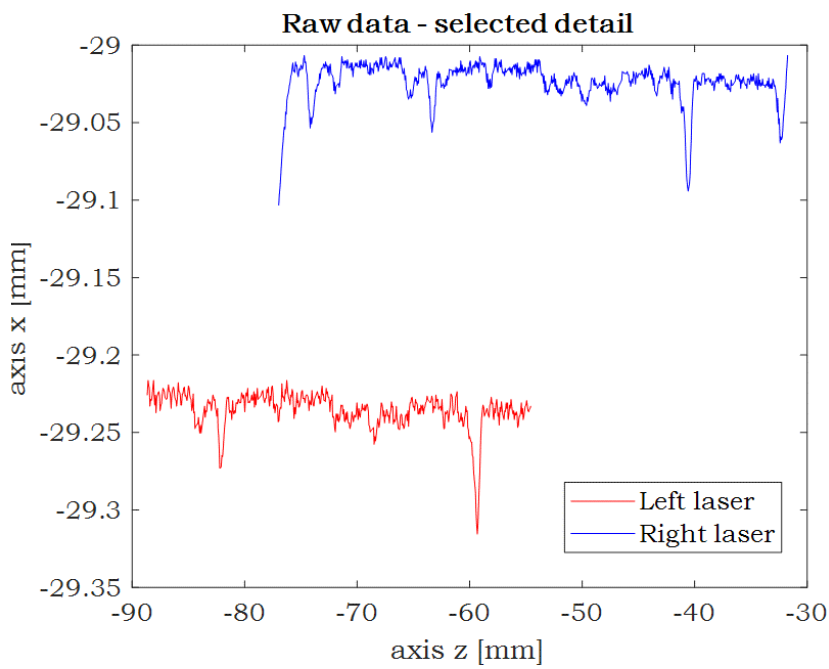

Fig. 7: Selected data detail

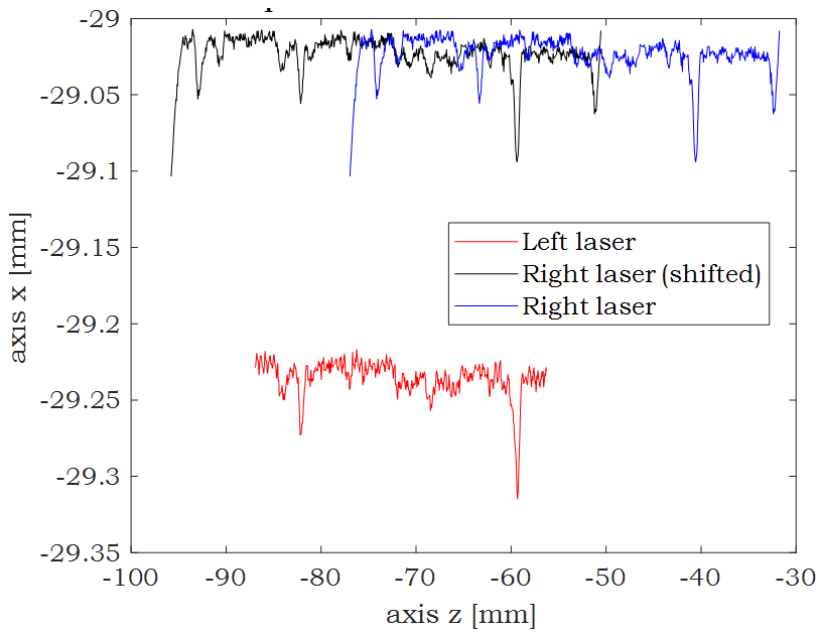

Fig. 9: Implementation of the shift in the axis $z$

We picked only the middle part of filtered data to obtain a shift, detail of signals is in Figure 7. Signals are shifted approximately 20 millimetres in the horizontal axis $Z$ and by 0.75 millimetres in the vertical axis $x$. The highest peak of the cross-correlation has occurred for the shift of minus 520 samples $(-18.825$ 
millimetres). The part of the cross-correlation function, where the whole signal from the left laser is included in the signal from the right sensor is plotted as a black line in Figure 9.

The implementation of the computed shift is illustrated in Figure 9. The final step is to align signals vertically using average values. From the right laser' signal was subtracted the difference of average values from both signals.

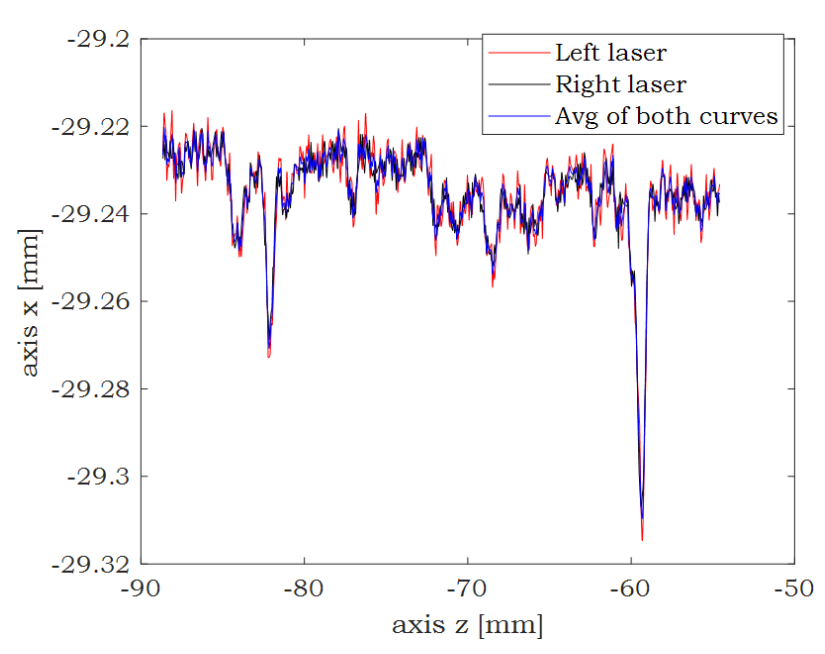

Fig. 10: Signals aligned in both axis
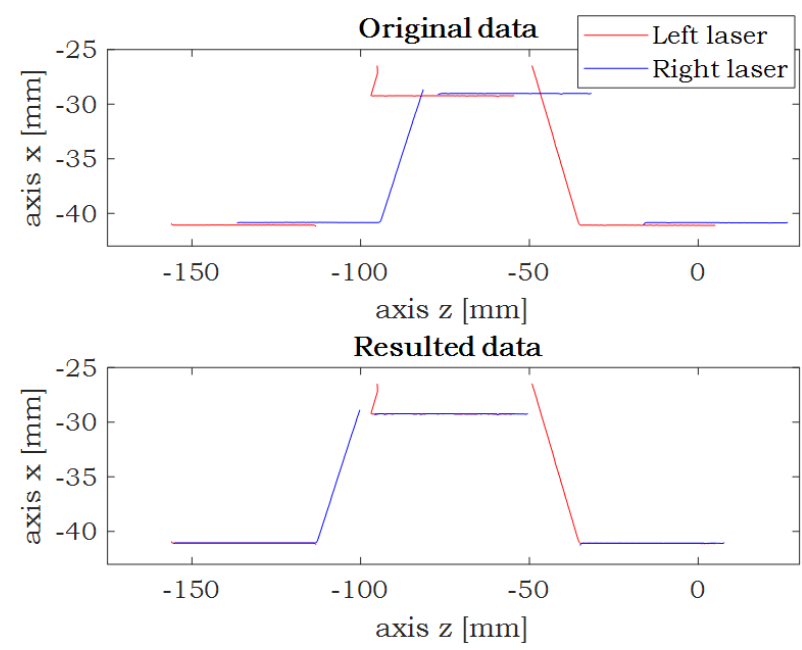

Fig. 11:Original data and shifted data

\section{Conclusion}

This paper presented usefulness of cross-correlation function for finding similar parts in two signals. This was presented in datasets acquire using a pair of optical laser micrometers. As both signals were situated in different positions acquired data were not aligned properly. In order to find a shift between these signals: basic filtration using the slope criterium of both signals to detect horizontal flat areas of signals was implemented. After that minor parts of the signals were selected the cross-correlation function was used to obtain an optimal shift in horizontal axis. The shifting of the vertical axis was evaluated using an average value of common parts of used signals. Result offset for both axes was applied to raw data and data were successfully overlaid.

\section{Acknowledgment}

This work was supported by the European Regional Development Fund in the Research Centre of Advanced Mechatronic Systems project, CZ.02.1.01/0.0/0.0/16_019/0000867 within the Operational Programme Research, Development and Education and the project SP2019/51 Applied Research in the Area of Machine and Process Control supported by the Ministry of Education, Youth and Sports.

\section{References}

Jones R. V. The Optical Micrometer [Article] // Optical Engineering. - June 1976. - 3 : Vol. 15. - p. 247.

Prieur Damien [et al.] Influence of the Microstructure on the U1-yAmyO2-x ( $y=0.1 ; 0.15)$ Pellet Macroscopic Swelling [Article] // Advances in Science and Technology. - [s.l.] : Trans Tech Publications, 2010. - Vol. 73. pp. 104-108.

Riftek Sensors \& Instruments Optical Micrometer RF656 Series User's Manual [Book]. - Minsk : [s.n.], 2018.

Smutný Lubomír, Mahdal Miroslav and Škuta Jaromír Smart sensors with PC connection in wireless networks [Article] // Proceedings of the 13th WSEAS International Conference on Computers - Held as part of the 13th WSEAS CSCC Multiconference2009. - Rodos : [s.n.], 2009. - 978-960474099-4.

Widmaier Thomas, Kuosmanen Petri Olavi and Juhanko Jari Measurement of Guideway Alignment of an on-Site Grinding Machine [Conference] // 56th International Scientific Colloquium. - 2011. 